\title{
Analysis of the erosive effect of different dietary substances and medications
}

\author{
Adrian Lussi*, Brigitte Megert, Robert Peter Shellis and Xiaojie Wang \\ Department of Preventive, Restorative and Pediatric Dentistry, School of Dental Medicine, University of Bern, Freiburgstrasse \\ 7, CH-3010 Bern, Switzerland \\ (Received 15 October 2010 - Revised 15 April 2011 - Accepted 15 April 2011 - First published online 30 June 2011)
}

\begin{abstract}
Excessive consumption of acidic drinks and foods contributes to tooth erosion. The aims of the present in vitro study were twofold: (1) to assess the erosive potential of different dietary substances and medications; (2) to determine the chemical properties with an impact on the erosive potential. We selected sixty agents: soft drinks, an energy drink, sports drinks, alcoholic drinks, juice, fruit, mineral water, yogurt, tea, coffee, salad dressing and medications. The erosive potential of the tested agents was quantified as the changes in surface hardness $(\Delta \mathrm{SH})$ of enamel specimens within the first $2 \mathrm{~min}\left(\Delta \mathrm{SH}_{2-0}=\mathrm{SH}_{2 \min }-\mathrm{SH}_{\text {baseline }}\right)$ and the second 2 min exposure $\left(\Delta \mathrm{SH}_{4-2}=\mathrm{SH}_{4 \mathrm{~min}}-\right.$ $\mathrm{SH}_{2}$ min). To characterise these agents, various chemical properties, e.g. $\mathrm{pH}$, concentrations of $\mathrm{Ca}, \mathrm{P}_{\mathrm{i}}$ and $\mathrm{F}$, titratable acidity to $\mathrm{pH} 7 \cdot 0$ and buffering capacity at the original $\mathrm{pH}$ value $(\beta)$, as well as degree of saturation $(\mathrm{p} K-\mathrm{p} I)$ with respect to hydroxyapatite (HAP) and fluorapatite (FAP), were determined. Erosive challenge caused a statistically significant reduction in SH for all agents except for coffee, some medications and alcoholic drinks, and non-flavoured mineral waters, teas and yogurts $(P<0 \cdot 01)$. By multiple linear regression analysis, $52 \%$ of the variation in $\Delta \mathrm{SH}$ after $2 \mathrm{~min}$ and $61 \%$ after $4 \mathrm{~min}$ immersion were explained by $\mathrm{pH}, \beta$ and concentrations of $\mathrm{F}$ and $\mathrm{Ca}(P<0.05)$. $\mathrm{pH}$ was the variable with the highest impact in multiple regression and bivariate correlation analyses. Furthermore, a high bivariate correlation was also obtained between $(\mathrm{p} K-\mathrm{p} I)_{\mathrm{HAP}},(\mathrm{p} K-\mathrm{p} I)_{\mathrm{FAP}}$ and $\Delta \mathrm{SH}$.
\end{abstract}

\section{Key words: Tooth erosion: Erosive potential: Dietary substances: Medications: Chemical properties: Hardness}

There is increasing evidence, from in vitro and in situ studies, that the excessive consumption of acidic drinks and foods poses a risk to dental hard tissues ${ }^{(1-8)}$. Tooth surface is softened in the early stage, and subsequently bulk material is dissolved, layer by layer, from the tooth surface. This type of tooth wear is defined as tooth erosion and is caused by acids (extrinsic and intrinsic) or chelating agents not involving bacterial action. There is a trend towards the increased consumption of acidic drinks and foods. In 2007, the worldwide annual consumption of soft drinks reached 552 billion litres, the equivalent of just under 83 litres/person per year, and this is projected to increase to 95 litres/person per year by 2012. However, the figure had already reached an average of 212 litres/person per year in the USA in $2009^{(9)}$. To decrease extrinsic erosive tooth wear, the emphasis should be on preventive strategies that mainly aim at reducing the exposure of teeth to potentially erosive agents.

As a prerequisite, it is essential for medical personnel and patients to have a thorough knowledge of the erosive potential of popular dietary substances. In the past several decades, studies investigating the erosive potential of different dietary substances and medications have been performed in different countries ${ }^{(3-8,10)}$. A wide range of drinks, foods and medications, such as soft drinks, sports drinks, juices, salad dressings, candies, herbal teas, alcoholic drinks, vinegar, vitamin $\mathrm{C}$ tablets etc., were recognised to be associated with the increase in erosion. Normally, soft drinks are mainly composed of filtered water, artificial additives and refined sugar. Thus, they offer limited nutritional benefit, but energy. Sports drinks, which are designed to replenish fluids lost during activity, typically contain water, electrolytes and sugar. Energy drinks are basically soft drinks that contain some forms of vitamins and other chemicals that boost energy for a very short span.

Various chemical properties of a potentially erosive agent, such as $\mathrm{pH}$ value, titratable acidity, buffering capacity, the concentrations of $\mathrm{Ca}, \mathrm{P}_{\mathrm{i}}$ and $\mathrm{F}$, have been identified in the literature to be potentially important in determining the erosive potential $^{(4,5,7,11,12)}$. However, to the best of our knowledge, no thorough analyses of the effects of a wide range of erosive agents have been undertaken. Buffering capacity is associated with the undissociated acid in a solution, and maintains the $\mathrm{H}^{+}$concentration and driving force for demineralisation at the site of dissolution ${ }^{(13,14)}$. The greater the buffering capacity

Abbreviations: FAP, fluorapatite; HAP, hydroxyapatite; $\mathrm{p} K-\mathrm{p} I$, degree of saturation; $\Delta \mathrm{SH}$, changes in surface hardness; SH, surface hardness. 
of the solution, the longer it will take for saliva to neutralise the acid, and as a result the more tooth mineral may be dissolved before a safe $\mathrm{pH}$ value is reached and the dissolution ceases. It is important to distinguish buffering capacity from titratable acidity. The latter measures total available $\mathrm{H}^{+}$over a wide range of $\mathrm{pH}$ values, whereas the former is defined at a certain $\mathrm{pH}$ value.

The aims of the present in vitro study were twofold: (1) to evaluate the erosive potential of different drinks, foods and medications; (2) to determine those chemical properties that have an impact on the erosive potential.

\section{Materials and methods}

\section{Preparation of enamel specimens}

From a pool of extracted teeth, six hundred caries-free human premolars with no cracks on the buccal sites as viewed under a stereomicroscope were selected. After the crowns of all teeth were separated from the roots, the buccal sites were ground flat under water-cooling on a LaboPol-21 rotating polishing machine (Struers, Ballerup, Denmark) as follows: groups of five enamel slabs were embedded into one resin disk (Paladur, Bad Homburg, Germany) in two planar parallel molds. Once the hardening process was complete, the thinner mold $(200 \mu \mathrm{m}$ thick) was removed. The outer $200 \mu \mathrm{m}$ of enamel were ground away with a silicon carbide paper disc of $18 \mu \mathrm{m}$ grade. Thereafter, the exposed buccal sides of enamel slabs in the thicker mold ( $7 \mathrm{~mm}$ thick) were serially polished on the polishing machine under constant cooling with silicon carbide paper discs of $8 \mu \mathrm{m}$ grade for $30 \mathrm{~s}$ and with $5 \mu \mathrm{m}$ grade for $1 \mathrm{~min}$. Then, after being taken out of the molds, the embedded resin disks, each containing five enamel slabs, were polished for $1 \mathrm{~min}$ with $3 \mu \mathrm{m}$ diamond abrasive on DP-Mol polishing cloth (Struers). After each polishing step, the resin disks were rinsed and sonicated for $2 \mathrm{~min}$ in tap water. These preparation steps wore away $200 \mu \mathrm{m}$ enamel substance in the centre of the window. Then all the resin disks with embedded enamel slabs were stored in a saturated mineral sol-

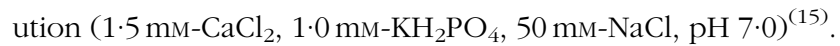

\section{Tested dietary substances and medications}

In the present study, sixty popular drinks, foods and medications in Switzerland were included (Table 1). According to their constituents and applications, these agents were divided into twelve groups: soft drinks, an energy drink, sports drinks, alcoholic drinks, juice, fresh fruit, mineral water, yogurt, tea, coffee, salad dressing and medications. Immediately before the experiment started, the fruits were crushed, and the pulps and seeds were removed by centrifugation; medication tablets and powders were dissolved in tap water according to the suggestions of the manufacturers.

\section{Chemical analysis of tested agents}

The $\mathrm{pH}$ value and the amount of base needed to raise the $\mathrm{pH}$ to $7 \cdot 0$ (titratable acidity) were measured with a titrator (Toledo
DL 53, Mettler Toledo, Electrode DG 101-SC, Software: LabX pro, Schwerzenbach, Switzerland). To measure titratable acidity, $10 \mathrm{~g}$ of each drink or solution were titrated with $0.5 \mathrm{M}-\mathrm{NaOH}$ in steps of $0.02 \mathrm{ml}$ at a temperature of $30^{\circ} \mathrm{C}$. The buffering capacity $(\beta)$ was calculated by using the following equation: $\beta=-\Delta C / \Delta \mathrm{pH}$, where $\Delta C$ is the amount of base used and $\Delta \mathrm{pH}$ is the change in $\mathrm{pH}$ caused by the addition of the base. In the present study, the buffering capacity at the original $\mathrm{pH}$ of the tested products was calculated.

All the tested agents were further analysed for Ca by standard atomic absorption using an atomic absorption spectrometer with an air/acetylene flame. Lanthanum was added to all the products and standards to suppress $\mathrm{P}_{\mathrm{i}}$ interference. Total $P_{i}$ concentration was analysed by the ammonium molybdate method of Chen et al. ${ }^{(16)}$. F concentration was determined using a F ion-specific electrode (Orion 960900, Boston, MA, USA). Before F measurement, all products and standard solutions were mixed with total ionic strength adjustment buffer (TISAB). The concentrations of $\mathrm{Ca}$ and $\mathrm{P}_{\mathrm{i}}$ are expressed in $\mathrm{mmol} / \mathrm{l}$ and those of $\mathrm{F}$ in $\mathrm{mg} / \mathrm{l}$.

The degree of saturation $(\mathrm{p} K-\mathrm{p} I)$ with respect to hydroxyapatite (HAP) and fluorapatite (FAP) was calculated from the $\mathrm{pH}$ and the concentrations of $\mathrm{Ca}, \mathrm{P}_{\mathrm{i}}$ and $\mathrm{F}$ using a computer program ${ }^{(17)}$. This program assumes a solubility product for HAP of $10^{-58 \cdot 5}$ and for FAP $10^{-596(18,19)}$.

Before the experiment, carbonated drinks were degassed by stirring at room temperature to avoid the adherence of bubbles to the enamel surface, which will affect the chemical analyses and hardness measurements. The concentrations of $\mathrm{Ca}, \mathrm{P}_{\mathrm{i}}$ and $\mathrm{F}$, the $\mathrm{pH}$ and the titratable acidity were measured in duplicate, and for further calculations of buffering capacity and $\mathrm{p} K-\mathrm{p} I$, the mean was determined.

\section{Surface hardness measurement}

Surface hardness (SH) of the enamel specimens was determined with a Vickers diamond under a pressure of $50 \mathrm{mN}$ for 15 s (Fischerscope HM 2000 XYp; Helmut Fischer, Hünenberg, Switzerland). A total of six baseline indentations were made at intervals of $70 \mu \mathrm{m}$. Further indentations next to the previous indentations were made following the experimental procedure. Vickers hardness was calculated from the dimensions of the indentations. The load resolution was $\leq 0.04 \mathrm{mN}$ and the indentation depth was $600 \mathrm{~nm}$ for sound enamel and $<1000 \mathrm{~nm}$ for most softened specimens. The device allowed fully automatic measurements using a programmable $\mathrm{x}, \mathrm{y}$ stage. The WIN-HCU software calculated and illustrated $\mathrm{SH}$.

\section{Study design}

After polishing the exposed enamel surface of resin disks (five enamel slabs each) with a $3 \mu \mathrm{m}$ diamond abrasive, six baseline indentations per specimen were made and measured. The mean $\mathrm{SH}$ for each resin disk, i.e. the average $\mathrm{SH}$ of five enamel samples, was then calculated. According to the $\mathrm{SH}$ distribution, two disks, with a total of ten enamel samples, were assigned to one of sixty groups. Thus, the average $\mathrm{SH}$ of each pair of disks was similar. Just before the experimental 
WS British Journal of Nutrition

Table 1. Basic information and various chemical parameters of the tested agents, e.g. $\mathrm{pH}$ value, titratable acidity to $\mathrm{pH} 7 \cdot 0$, buffering capacity at the $\mathrm{pH}$ value, $\mathrm{Ca}$, $\mathrm{P}_{\mathrm{i}}$, and $\mathrm{F}$ concentrations, degree of saturation with respect to hydroxyapatite and fluorapatite

\begin{tabular}{|c|c|c|c|c|c|c|c|c|c|c|c|}
\hline Tested agents & $\begin{array}{l}\text { Brand } \\
\text { name/producer }\end{array}$ & Flavour & $\begin{array}{l}\text { Erosion-related } \\
\text { ingredients }\end{array}$ & $\mathrm{pH}$ & $\begin{array}{c}\mathrm{mmol} \mathrm{OH}^{-} / \mathrm{l} \\
\text { to } \mathrm{pH} 7 \cdot 0\end{array}$ & $\begin{array}{l}\beta(\mathrm{mmol} / \mathrm{l} \\
\times \mathrm{pH})\end{array}$ & $\underset{(\mathrm{mmol} / \mathrm{l})}{[\mathrm{Ca}]}$ & $\begin{array}{c}{\left[\mathrm{P}_{\mathrm{i}}\right]} \\
(\mathrm{mmol} / \mathrm{l})\end{array}$ & $\begin{array}{c}{[\mathrm{F}]} \\
(\mathrm{mg} / \mathrm{l})\end{array}$ & $(p K-p /$ HAP & $(\mathrm{p} K-\mathrm{p})_{\mathrm{FAP}}$ \\
\hline \multicolumn{12}{|l|}{ Soft drinks } \\
\hline $\begin{array}{l}\text { Carpe Diem } \\
\text { Kombucha fresh }\end{array}$ & $\begin{array}{l}\text { Carpe Diem/Carpe } \\
\text { Diem GmbH \& Co KG }\end{array}$ & NA & $\begin{array}{l}\text { Herbal tea extract and } \\
\text { carbonic acid }\end{array}$ & 3.00 & $39 \cdot 0$ & $17 \cdot 6$ & $3 \cdot 30$ & 0.07 & 0.39 & $-19 \cdot 0$ & $-12 \cdot 0$ \\
\hline Coca-Cola & $\begin{array}{l}\text { Coca-Cola/Coca-Cola } \\
\text { company }\end{array}$ & Cola & $\begin{array}{l}\text { Phosphoric acid } \\
\text { and flavours }\end{array}$ & $2 \cdot 45$ & $17 \cdot 5$ & 9.6 & 1.08 & $5 \cdot 04$ & 0.22 & $-20 \cdot 0$ & $-13 \cdot 1$ \\
\hline Coca-Cola light & $\begin{array}{l}\text { Coca-Cola/Coca-Cola } \\
\text { Company }\end{array}$ & Cola & $\begin{array}{l}\text { Phosphoric acid, citric } \\
\text { acid and flavours }\end{array}$ & $2 \cdot 60$ & $19 \cdot 0$ & $7 \cdot 3$ & 0.82 & 4.85 & 0.22 & $-19 \cdot 4$ & $-12 \cdot 5$ \\
\hline $\begin{array}{l}\text { Fanta regular } \\
\text { orange }\end{array}$ & $\begin{array}{l}\text { Fanta/Coca-Cola } \\
\text { Company }\end{array}$ & Orange & $\begin{array}{l}\text { Orange fruit, citric acid, } \\
\text { flavours and } \\
\text { acidity regulator }\end{array}$ & $2 \cdot 67$ & 52.5 & $15 \cdot 8$ & 0.48 & 0.08 & 0.04 & $-25 \cdot 2$ & $-19 \cdot 1$ \\
\hline Ice tea classic & $\begin{array}{l}\text { n/a /Coop } \\
\text { (supermarket in } \\
\text { Switzerland) }\end{array}$ & NA & Black tea extract & $2 \cdot 94$ & $26 \cdot 5$ & $15 \cdot 0$ & 0.45 & 0.04 & 0.76 & -24.2 & -16.9 \\
\hline Ice tea lemon & Lipton/Unilever & Lemon & $\begin{array}{l}\text { Black tea extract and } \\
\text { lemon juice }\end{array}$ & 3.03 & $24 \cdot 0$ & $9 \cdot 4$ & $0 \cdot 18$ & $0 \cdot 12$ & 0.58 & $-24 \cdot 0$ & $-16 \cdot 8$ \\
\hline Ice tea peach & Lipton/Unilever & Peach & $\begin{array}{l}\text { Black tea extract and } \\
\text { peach juice }\end{array}$ & $2 \cdot 94$ & $21 \cdot 5$ & 8.5 & 0.12 & 0.15 & 0.53 & $-25 \cdot 2$ & $-18 \cdot 0$ \\
\hline Pepsi Cola & $\begin{array}{l}\text { Pepsi Cola/Pepsi } \\
\text { Company, Inc. }\end{array}$ & Cola & $\begin{array}{l}\text { Phosphoric acid, citric } \\
\text { acid and flavours }\end{array}$ & $2 \cdot 39$ & $19 \cdot 0$ & $11 \cdot 7$ & 0.33 & 4.93 & 0.04 & $-23 \cdot 0$ & $-16 \cdot 9$ \\
\hline Pepsi Cola light & $\begin{array}{l}\text { Pepsi Cola/Pepsi } \\
\text { Company, Inc. }\end{array}$ & Cola & $\begin{array}{l}\text { Phosphoric acid, citric acid } \\
\text { and flavours }\end{array}$ & $2 \cdot 77$ & $15 \cdot 0$ & $7 \cdot 4$ & 0.29 & $4 \cdot 68$ & 0.04 & $-20 \cdot 4$ & $-14 \cdot 3$ \\
\hline Rivella blue & $\begin{array}{l}\text { Rivella/Rivella } \\
\text { International AG }\end{array}$ & NA & $\begin{array}{l}\text { Milk serum, carbonic acid, } \\
\text { citric acid } \\
\text { and flavours }\end{array}$ & $3 \cdot 31$ & 38.0 & $37 \cdot 9$ & 4.00 & $2 \cdot 17$ & 0.08 & $-12 \cdot 0$ & -5.9 \\
\hline Rivella green & $\begin{array}{l}\text { Rivella/Rivella } \\
\text { International AG }\end{array}$ & Green tea & $\begin{array}{l}\text { Milk serum, green tea } \\
\text { extract, carbonic } \\
\text { acid, citric acid } \\
\text { and flavours }\end{array}$ & $3 \cdot 22$ & 44 & 37.5 & $3 \cdot 30$ & $2 \cdot 41$ & 0.09 & $-12 \cdot 9$ & -6.6 \\
\hline Rivella red & $\begin{array}{l}\text { Rivella/Rivella } \\
\text { International AG }\end{array}$ & NA & $\begin{array}{l}\text { Milk serum, carbonic acid, } \\
\text { citric acid and flavours }\end{array}$ & $3 \cdot 28$ & 41.5 & $35 \cdot 4$ & $3 \cdot 13$ & $2 \cdot 28$ & 0.08 & $-12 \cdot 6$ & -6.5 \\
\hline Sinalco & $\begin{array}{l}\text { Sinalco/Sinalco } \\
\text { International }\end{array}$ & Orange & $\begin{array}{l}\text { Orange juice, carbonic acid, } \\
\text { citric acid, mandarin juice, } \\
\text { lemon juice, ascorbic acid } \\
\text { and flavours }\end{array}$ & $3 \cdot 12$ & $36 \cdot 0$ & $10 \cdot 1$ & $1 \cdot 14$ & $0 \cdot 10$ & 0.06 & $-19 \cdot 7$ & -13.5 \\
\hline Sprite & $\begin{array}{l}\text { Sprite/Coca-Cola } \\
\text { Company }\end{array}$ & Sour & $\begin{array}{l}\text { Carbonic acid, citric acid, } \\
\text { acidity regulator } \\
\text { and flavours }\end{array}$ & 2.54 & $39 \cdot 0$ & $15 \cdot 0$ & 0.30 & 0.02 & 0.02 & $-28 \cdot 8$ & $-23 \cdot 0$ \\
\hline \multicolumn{12}{|l|}{ Energy drink } \\
\hline Red Bull & $\begin{array}{l}\text { Red Bull/Red } \\
\text { Bull GmbH }\end{array}$ & NA & Taurine and $B$ vitamins & 3.30 & $98 \cdot 0$ & $45 \cdot 5$ & 1.94 & $<0.01$ & 0.11 & $-26 \cdot 4$ & $-20 \cdot 1$ \\
\hline \multicolumn{12}{|l|}{ Sports drinks } \\
\hline Gatorade & $\begin{array}{l}\text { Gatorade/Pepsi } \\
\text { Company, Inc. }\end{array}$ & Sour & Citric acid and flavours & $3 \cdot 17$ & $46 \cdot 0$ & $21 \cdot 8$ & 0.13 & 2.98 & 0.05 & $-19 \cdot 7$ & $-13 \cdot 7$ \\
\hline Isostar & $\begin{array}{l}\text { Isostar/Novartis } \\
\text { International AG }\end{array}$ & NA & $\begin{array}{l}\text { Citric acid, flavours and } \\
\text { ascorbic acid }\end{array}$ & 3.87 & $56 \cdot 5$ & $52 \cdot 8$ & $8 \cdot 20$ & 4.49 & 0.11 & -5.9 & -0.1 \\
\hline Powerade & $\begin{array}{l}\text { Powerade/Coca-Cola } \\
\text { Company }\end{array}$ & Lemon & $\begin{array}{l}\text { Malic acid, vitamins } \\
\mathrm{B}_{2} \text { and } \mathrm{B}_{6}\end{array}$ & 3.74 & $43 \cdot 0$ & $18 \cdot 0$ & 0.25 & $<0.01$ & 0.21 & -22.5 & $-16 \cdot 2$ \\
\hline \multicolumn{12}{|c|}{$D_{2}$ dnu $D_{6}$} \\
\hline Apple juice & $\begin{array}{l}\text { Ramseier/Ramseier } \\
\text { Suisse AG }\end{array}$ & Premium & $\begin{array}{l}\text { Apple juice and } \\
\text { pear juice }\end{array}$ & 3.41 & $72 \cdot 0$ & $43 \cdot 6$ & 1.96 & 1.66 & 0.06 & $-13 \cdot 0$ & $-7 \cdot 0$ \\
\hline
\end{tabular}


WS British Journal of Nutrition

Table 1. Continued

\begin{tabular}{|c|c|c|c|c|c|c|c|c|c|c|c|}
\hline Tested agents & $\begin{array}{l}\text { Brand } \\
\text { name/producer }\end{array}$ & Flavour & $\begin{array}{l}\text { Erosion-related } \\
\text { ingredients }\end{array}$ & $\mathrm{pH}$ & $\begin{array}{c}\mathrm{mmol} \mathrm{OH}^{-} / \mathrm{l} \\
\text { to } \mathrm{pH} 7.0\end{array}$ & $\begin{array}{l}\beta(\mathrm{mmol} / \mathrm{l} \\
\times \mathrm{pH})\end{array}$ & $\begin{array}{c}{[\mathrm{Ca}]} \\
(\mathrm{mmol} / \mathrm{l})\end{array}$ & $\begin{array}{c}{\left[\mathrm{P}_{\mathrm{i}}\right]} \\
(\mathrm{mmol} / \mathrm{l})\end{array}$ & $\begin{array}{c}{[\mathrm{F}]} \\
(\mathrm{mg} / \mathrm{l})\end{array}$ & $(\mathrm{p} K-\mathrm{p} /)_{\mathrm{HAP}}$ & $(p K-p)_{F A P}$ \\
\hline Carrot juice & Biotta/Biotta AG & Carrot & $\begin{array}{l}\text { Carrot juice, orange juice, } \\
\text { agave juice, } \\
\text { lemon juice and } \\
\text { ascorbic acid }\end{array}$ & $4 \cdot 16$ & $70 \cdot 5$ & $55 \cdot 7$ & 4.40 & $1 \cdot 20$ & 0.04 & $-6 \cdot 6$ & $-1 \cdot 4$ \\
\hline Grapefruit juice & n/a /Coop & Grape fruit & Grapefruit juice & 3.15 & $168 \cdot 5$ & 71.4 & $2 \cdot 30$ & $2 \cdot 17$ & 0.03 & $-14 \cdot 2$ & -8.4 \\
\hline $\begin{array}{l}\text { Orange juice, } \\
\text { Del Monte }\end{array}$ & $\begin{array}{l}\text { n/a /Migros } \\
\quad \text { (supermarket } \\
\text { in Switzerland) }\end{array}$ & Orange & Orange juice & 3.74 & $108 \cdot 0$ & $66 \cdot 7$ & $2 \cdot 38$ & 2.36 & 0.03 & -9.8 & -4.5 \\
\hline $\begin{array}{c}\text { Orange juice, } \\
\text { Hohes C }\end{array}$ & Hohes C/Eckes AG & Orange & Orange juice & 3.56 & $121 \cdot 0$ & $62 \cdot 5$ & 1.98 & 2.57 & 0.03 & $-11 \cdot 4$ & $-5 \cdot 8$ \\
\hline \multicolumn{12}{|l|}{ Fruit } \\
\hline Apricot & NA & Apricot & NA & 3.25 & $317 \cdot 0$ & $125 \cdot 0$ & $1 \cdot 20$ & 5.95 & 0.02 & -13.6 & -8.0 \\
\hline Kiwi & NA & Kiwi & NA & 3.25 & 206.5 & 142.9 & 3.35 & 4.47 & 0.02 & -11.9 & -6.3 \\
\hline \multirow{2}{*}{\multicolumn{12}{|c|}{ Alcoholic drinks }} \\
\hline & & & & & & & & & & & \\
\hline $\begin{array}{l}\text { Bacardi Breezer } \\
\text { orange }\end{array}$ & $\begin{array}{l}\text { Bacardi/Bacardi \& } \\
\text { Company Limited }\end{array}$ & Orange & $\begin{array}{l}4.4 \% \text { alcohol and } \\
\text { orange juice }\end{array}$ & $3 \cdot 16$ & $60 \cdot 0$ & $26 \cdot 1$ & $0 \cdot 19$ & 0.14 & 0.03 & $-22 \cdot 5$ & $-16 \cdot 7$ \\
\hline Cynar & Cynar/Campari Gruppo & NA & $16.5 \%$ alcohol & 4.00 & $6 \cdot 0$ & $5 \cdot 8$ & $2 \cdot 01$ & 0.13 & 0.07 & $-12 \cdot 0$ & -6.5 \\
\hline Beer, Carlsberg & $\begin{array}{l}\text { Carlsberg/ Carlsberg } \\
\text { Group }\end{array}$ & NA & $5 \cdot 0 \%$ alcohol & $4 \cdot 20$ & $17 \cdot 5$ & $8 \cdot 3$ & 0.74 & 5.65 & 0.74 & $-7 \cdot 9$ & -1.5 \\
\hline Beer, Eichhof & $\begin{array}{c}\text { Eichhof/Eichhof } \\
\text { Getränke AG }\end{array}$ & NA & $4.9 \%$ alcohol & 4.07 & $18 \cdot 0$ & $8 \cdot 1$ & 1.94 & $9 \cdot 30$ & 0.06 & $-6 \cdot 3$ & -0.9 \\
\hline Champagner & Freixenet/Grupo Freixenet & NA & $12.0 \%$ alcohol & 2.99 & $78 \cdot 0$ & $35 \cdot 8$ & 1.90 & 1.98 & 0.26 & $-15 \cdot 9$ & $-9 \cdot 0$ \\
\hline Red wine, Collivo & Collivo/Italia & NA & $13.0 \%$ alcohol & 3.43 & $76 \cdot 0$ & 54.4 & 1.25 & 4.69 & 0.07 & -12.5 & -6.4 \\
\hline $\begin{array}{l}\text { Red wine, } \\
\text { Montagne }\end{array}$ & Montagne/France & NA & $11.7 \%$ alcohol & 3.68 & $63 \cdot 0$ & $46 \cdot 5$ & 1.68 & $2 \cdot 79$ & 0.11 & $-10 \cdot 7$ & $-4 \cdot 7$ \\
\hline White wine & La Côte/France & NA & $12 \cdot 1 \%$ alcohol & 3.60 & $53 \cdot 0$ & $50 \cdot 0$ & 1.30 & 4.42 & 0.27 & $-11 \cdot 3$ & $-4 \cdot 8$ \\
\hline Smirnoff ice vodka & Smirnoff/Diageo plc & Lemon & $\begin{array}{l}40.0 \% \text { alcohol } \\
\text { and lemon juice }\end{array}$ & 3.07 & $50 \cdot 0$ & $18 \cdot 2$ & 0.18 & 6.54 & 0.12 & $-18 \cdot 8$ & $-12 \cdot 4$ \\
\hline \multicolumn{12}{|l|}{ Medications } \\
\hline Alca-C fizzy tablet & $\begin{array}{l}\text { Alca-C/Novartis } \\
\text { Consumer } \\
\text { Health Schweiz AG }\end{array}$ & Orange & $\begin{array}{l}\text { Acetylsalicylic acid } \\
\text { and ascorbic acid }\end{array}$ & $4 \cdot 20$ & 53.0 & $45 \cdot 5$ & 9.03 & 0.02 & 0.07 & $-10 \cdot 2$ & $-4 \cdot 8$ \\
\hline Alcacyl 500 & $\begin{array}{l}\text { Alcacyl 500/Novartis } \\
\text { Consumer Health } \\
\text { Schweiz AG }\end{array}$ & Sour & Acetylsalicylic acid & $6 \cdot 93$ & 0.5 & $3 \cdot 7$ & 1.89 & $<0.01$ & 0.07 & 0.4 & $3 \cdot 1$ \\
\hline $\begin{array}{l}\text { Alka-Seltzer fizzy } \\
\text { tablet }\end{array}$ & $\begin{array}{l}\text { Alka-Seltzer/Bayer } \\
\text { (Schweiz) AG }\end{array}$ & Sour & $\begin{array}{l}\text { Acetylsalicylic acid } \\
\text { and citric acid }\end{array}$ & $6 \cdot 23$ & $14 \cdot 0$ & $24 \cdot 9$ & 2.06 & 0.03 & 0.08 & 1.5 & $5 \cdot 0$ \\
\hline $\begin{array}{l}\text { Aspirine-C fizzy } \\
\text { tablet }\end{array}$ & $\begin{array}{l}\text { Aspirine-C/Bayer } \\
\text { (Schweiz) AG }\end{array}$ & Sour & $\begin{array}{l}\text { Acetylsalicylic acid } \\
\text { and vitamin C }\end{array}$ & $5 \cdot 51$ & $27 \cdot 5$ & $32 \cdot 8$ & $2 \cdot 04$ & $<0.01$ & 0.08 & $-5 \cdot 7$ & -1.5 \\
\hline $\begin{array}{l}\text { Berocca fizzy } \\
\text { tablet }\end{array}$ & $\begin{array}{l}\text { Berocca/Bayer } \\
\text { (Schweiz) AG }\end{array}$ & Orange & Vitamin C & $4 \cdot 24$ & 59.5 & $57 \cdot 2$ & $15 \cdot 20$ & 0.03 & 0.12 & -8.9 & $-3 \cdot 3$ \\
\hline $\begin{array}{r}\text { Fluimucil } 200 \\
\text { fizzy tablet }\end{array}$ & Zambon & Orange & $\begin{array}{l}\text { Tartaric acid, citric } \\
\text { acid monohydrate } \\
\text { and flavours }\end{array}$ & 4.71 & $19 \cdot 5$ & $13 \cdot 5$ & 1.98 & $<0.01$ & 0.06 & -12.5 & $-7 \cdot 6$ \\
\hline Neocitran & $\begin{array}{l}\text { Neocitran/Novartis } \\
\text { Consumer } \\
\text { Health Schweiz AG }\end{array}$ & Sour & Vitamin C & $2 \cdot 85$ & 73.5 & $27 \cdot 5$ & 4.63 & 1.58 & 0.09 & -15.5 & -0.9 \\
\hline
\end{tabular}


NS Bristh houma of Nurrition

Table 1. Continued

\begin{tabular}{|c|c|c|c|c|c|c|c|c|c|c|c|}
\hline Tested agents & $\begin{array}{l}\text { Brand } \\
\text { name/producer }\end{array}$ & Flavour & $\begin{array}{l}\text { Erosion-related } \\
\text { ingredients }\end{array}$ & $\mathrm{pH}$ & $\begin{array}{c}\mathrm{mmol} \mathrm{OH}^{-} / 1 \\
\text { to } \mathrm{pH} 7 \cdot 0\end{array}$ & $\begin{array}{l}\beta(\mathrm{mmol} / \mathrm{l} \\
\times \mathrm{pH})\end{array}$ & $\begin{array}{c}{[\mathrm{Ca}]} \\
(\mathrm{mmol} / \mathrm{l})\end{array}$ & $\begin{array}{c}{\left[\mathrm{P}_{\mathrm{i}}\right]} \\
(\mathrm{mmol} / \mathrm{l})\end{array}$ & $\begin{array}{c}{[\mathrm{F}]} \\
(\mathrm{mg} / \mathrm{l})\end{array}$ & $(p K-p /$ HAP & $(\mathrm{p} K-\mathrm{p})_{\mathrm{FAP}}$ \\
\hline $\begin{array}{l}\text { Vitamin C fizzy } \\
\text { tablet, } \\
\text { Actilife }\end{array}$ & Actilife/Migros & Orange & $\begin{array}{l}\text { Citric acid, vitamin C } \\
\text { and flavours }\end{array}$ & 3.86 & $93 \cdot 0$ & $58 \cdot 8$ & 1.90 & 0.03 & 0.06 & $-15 \cdot 0$ & -9.4 \\
\hline $\begin{array}{l}\text { Vitamin C fizzy } \\
\text { tablet, } \\
\text { Streuli }\end{array}$ & Streuli/Streuli Pharma AG & Sour & Vitamin C & 3.63 & $85 \cdot 0$ & $42 \cdot 6$ & $1 \cdot 78$ & $2 \cdot 01$ & 0.06 & -11.4 & $-5 \cdot 6$ \\
\hline Siccoral & $\begin{array}{l}\text { Siccoral/Drossa } \\
\text { Pharma GmbH }\end{array}$ & NA & NA & $5 \cdot 41$ & $2 \cdot 5$ & $2 \cdot 0$ & 0.15 & 0.12 & 0.03 & $-7 \cdot 4$ & $-3 \cdot 6$ \\
\hline \multicolumn{12}{|l|}{ Yogurt } \\
\hline Kiwi Tropicana & Hirz/Nestlé Switzerland & Kiwi & $\begin{array}{l}\text { Kiwi and } \\
\text { exotic fruits }\end{array}$ & 3.99 & 124.5 & $111 \cdot 1$ & $45 \cdot 83$ & 33.82 & 0.04 & 0 & $5 \cdot 3$ \\
\hline Nature & n/a /Migros & Milky & NA & 3.91 & $120 \cdot 0$ & 95.5 & 43.33 & 34.34 & 0.04 & -0.6 & 4.7 \\
\hline Slimline & n/a /Migros & Milky & NA & 4.03 & 133.5 & $100 \cdot 0$ & $56 \cdot 33$ & 38.74 & 0.03 & 0.8 & 5.9 \\
\hline Forest berries & n/a /Migros & Berries & Forest berries & 3.77 & 159.0 & $200 \cdot 0$ & $45 \cdot 50$ & $36 \cdot 81$ & 0.05 & -1.4 & $4 \cdot 1$ \\
\hline \multicolumn{12}{|l|}{ Mineral water } \\
\hline Henniez & Henniez/Nestlé Waters & NA & NA & 7.68 & N.A & $2 \cdot 0$ & 2.48 & $<0.01$ & $0 \cdot 10$ & $2 \cdot 4$ & 4.5 \\
\hline Henniez sparkling & Henniez/Nestlé Waters & NA & NA & $6 \cdot 13$ & 4.0 & 4.2 & $2 \cdot 40$ & $<0.01$ & 0.09 & $-6 \cdot 2$ & -2.6 \\
\hline Valser & $\begin{array}{l}\text { Valser/Valser } \\
\text { Mineralquellen AG }\end{array}$ & NA & NA & $5 \cdot 63$ & $12 \cdot 5$ & $10 \cdot 9$ & 9.93 & $<0.01$ & 0.60 & $-2 \cdot 8$ & $2 \cdot 1$ \\
\hline $\begin{array}{l}\text { Valser Viva } \\
\text { Lemon }\end{array}$ & $\begin{array}{l}\text { Valser/Valser } \\
\quad \text { Mineralquellen AG }\end{array}$ & $\begin{array}{l}\text { Lemon } \\
\quad \text { and herbs }\end{array}$ & $\begin{array}{l}\text { Lemon } \\
\quad \text { and herbs }\end{array}$ & 3.31 & $40 \cdot 0$ & $21 \cdot 1$ & $9 \cdot 75$ & 0.08 & 0.63 & $-14 \cdot 7$ & $-7 \cdot 6$ \\
\hline \multicolumn{12}{|l|}{ Tea } \\
\hline Rose hip & n/a /Migros & Rose hip & Rose hip & 3.15 & 19.5 & $19 \cdot 7$ & 2.65 & 0.42 & 0.05 & $-16 \cdot 0$ & $-10 \cdot 0$ \\
\hline Pepper mint & n/a /Migors & Pepper mint & Pepper mint & 7.51 & N.A & 2.9 & 1.93 & 0.35 & 0.05 & $11 \cdot 8$ & $13 \cdot 8$ \\
\hline Black tea & n/a /Coop & Black tea & Black tea & 6.59 & 1.5 & $2 \cdot 6$ & $1 \cdot 10$ & 0.27 & 1.63 & $5 \cdot 6$ & $10 \cdot 0$ \\
\hline Wild berries & Lipton/Unilever & Berries & $\begin{array}{l}\text { Hibiscus bloom, } \\
\text { apple, strawberry, } \\
\text { currant and } \\
\text { blackberry }\end{array}$ & 6.78 & 1.0 & $2 \cdot 6$ & $1 \cdot 10$ & 0.24 & 0.78 & $6 \cdot 6$ & $10 \cdot 5$ \\
\hline \multicolumn{12}{|l|}{ Coffee } \\
\hline Espresso & $\begin{array}{l}\text { Nestlé /Nestlé } \\
\text { Switzerland }\end{array}$ & Coffee & NA & $5 \cdot 82$ & 3.0 & $2 \cdot 2$ & 0.69 & 0.63 & 0.07 & 0.6 & 4.5 \\
\hline \multicolumn{12}{|l|}{ Salad dressing } \\
\hline $\begin{array}{l}\text { Thomy French } \\
\text { Classic }\end{array}$ & $\begin{array}{l}\text { Thomy/Nestlé } \\
\text { Switzerland }\end{array}$ & $\begin{array}{l}\text { Creamy } \\
\text { and sour }\end{array}$ & $\begin{array}{l}\text { Vinegar and } \\
\text { lemon juice }\end{array}$ & 4.04 & $141 \cdot 0$ & $111 \cdot 1$ & $20 \cdot 50$ & 0.46 & 0.11 & $-6 \cdot 1$ & -0.5 \\
\hline $\begin{array}{l}\text { Thomy French } \\
\text { Light }\end{array}$ & Thomy/Nestlé Switzerland & $\begin{array}{l}\text { Creamy } \\
\text { and sour }\end{array}$ & Vinegar & 3.85 & $145 \cdot 0$ & $100 \cdot 0$ & $40 \cdot 00$ & $1 \cdot 14$ & 0.11 & $-5 \cdot 3$ & 0.5 \\
\hline
\end{tabular}

*Titratable acidity, $\mathrm{mmol} \mathrm{OH}^{-1 /}$ to $\mathrm{pH} 7 \cdot 0 ; \beta$, buffering capacity at the $\mathrm{pH}$ value; $\mathrm{pK}-\mathrm{p} /$ with respect to HAP and $\mathrm{p} K-\mathrm{p} /$ with respect to FAP. 
procedures, the resin disks were further polished with a $1 \mu \mathrm{m}$ diamond abrasive for $1 \mathrm{~min}$ (LaboPol-6, DP-Mol Polishing, DP-Stick HQ; Struers, Copenhagen, Denmark), which assured the removal of the possible remnants from storage.

Before the erosive challenge, enamel specimens were immersed in $20 \mathrm{ml}$ of freshly collected human saliva for $3 \mathrm{~h}$ to form a salivary pellicle. The saliva, stimulated by paraffin wax (Fluka; Sigma-Aldrich Chemie GmbH, Munich, Germany), was collected in an ice-cooled tube from a single healthy donor at least $1 \mathrm{~h}$ after any intake of drink or food ${ }^{(20,21)}$. She gave informed consent, and saliva collection was performed in accordance with the protocol approved by the University of Bern (Bern, Switzerland). After being carefully rinsed with tap water for $50 \mathrm{~s}$, with deionised water for $10 \mathrm{~s}$ and then dried for $5 \mathrm{~s}$ with oil-free air, the $\mathrm{SH}$ baseline of the samples was measured. Afterwards, the resin disks with five enamel specimens each were individually placed in $60 \mathrm{ml}$ (or g) of the appropriate solution under constant agitation $(95 \mathrm{rpm})$ at $30^{\circ} \mathrm{C}$ (shaking bath Salvis; Renggli AG, Rotkreuz, Switzerland). After immersion for 2 and $4 \mathrm{~min}$, the resin disks were taken out of the solution, and the $\mathrm{SH}$ measurement was performed once again.

\section{Statistics}

Wilcoxon's signed rank tests were calculated in an attempt to compare the $\mathrm{SH}$ values before and after immersion. The relationship between the changes in $\mathrm{SH}(\Delta \mathrm{SH})$ within the first $2 \mathrm{~min}\left(\Delta \mathrm{SH}_{2-0}=\mathrm{SH}_{2 \text { min }}-\mathrm{SH}_{\text {baseline }}\right)$ and the second 2 min $\left(\Delta \mathrm{SH}_{4-2}=\mathrm{SH}_{4 \text { min }}-\mathrm{SH}_{2 \text { min }}\right)$ immersion (dependent variables) and $\mathrm{pH}$, buffering capacity, and $\mathrm{Ca}, \mathrm{P}_{\mathrm{i}}$ and $\mathrm{F}$ concentrations (independent variables) was investigated using multiple linear regression (backward selection) analyses. Only variables independent from each other were included. The $\mathrm{p} K-\mathrm{p} I$ and the titratable acidity were not eligible for inclusion. To assess the bivariate associations between different chemical properties and $\Delta \mathrm{SH}$ after 2 or $4 \mathrm{~min}$ exposure, Spearman's correlation coefficients were used. The statistical calculations were performed using SAS Enterprise Guide 4.1 software. The significance level was set at 0.01 in Wilcoxon's signed rank tests and at 0.05 in multiple linear regression and Spearman's correlation analyses.

\section{Results}

\section{Changes in the surface hardness of enamel}

In Table 2, Wilcoxon's signed rank tests revealed a significant reduction $(P<0 \cdot 01)$ of $\Delta \mathrm{SH}_{2-0}$ for soft drinks, sports drinks, the energy drink (Red Bull), juices (except for carrot juice), fruits and salad dressings. Except for Isostar (sports drink) and Thomy French Classic salad dressing, these substances presented a trend towards further decrease in $\Delta \mathrm{SH}_{4-2}$. On the contrary, no statistically significant change was found for coffee, most mineral waters, teas and yogurts in both $\Delta \mathrm{SH}_{2-0}$ and $\Delta \mathrm{SH}_{4-2}$. Exceptions were rose hip tea, forest berries yogurt and Valser Viva Lemon mineral water that had a similar erosive effect as soft drinks. A complicated erosive pattern was observed in the medication and alcoholic drink groups. For example, Alca-C, Alcacyl 500 and Berocca fizzy tablets did not induce a significant decrease in $\mathrm{SH}$, while the reduction was observable within the first 2 min for Aspirine-C fizzy tablet and within the second 2 min for Siccoral, Alka-Seltzer and Fluimucil 200 fizzy tablets. In the alcoholic drink group, by the end of the experiment, Cynar, Carlsberg beer and Montagne red wine did not produce any significant changes in SH of enamel specimens, whereas Eichhof beer demonstrated erosive potential within the second $2 \mathrm{~min}$. It is worth noting that as no adjustment for multiple testing was done, the present results can only be taken into exploratory consideration.

\section{Influence of different chemical properties on changes in surface hardness}

Table 1 also gives an overview of the chemical properties of all tested agents.

Coffee, teas (except for rose hip tea), mineral waters (except for Valser Viva Lemon mineral water) and some medications (Alcacyl 500, Alka-Seltzer and Aspirine-C fizzy tablets) had the highest $\mathrm{pH}$ values, above 5.5. The lowest $\mathrm{pH}$ values, varying between $2 \cdot 4$ and $3 \cdot 3$, were mostly found in the soft drinks and the energy drink (Red Bull).

The larger titratable acidity was found for fruits, salad dressings, yogurts as well as for grapefruit and orange juices $(>100 \mathrm{mmol} / \mathrm{l})$. The buffering capacity ranged from $2 \cdot 0$ to $200 \mathrm{mmol} / \mathrm{l} \times \mathrm{pH}$. The highest values were observed for yogurts, fruits (except for orange) and salad dressings $(>95 \mathrm{mmol} / 1 \times \mathrm{pH})$, the lowest values for Siccoral, Henniez mineral water, coffee and tea (except for rose hip tea) $(<3 \mathrm{mmol} / \mathrm{l} \times \mathrm{pH})$.

Yogurts contained the highest concentrations of $\mathrm{Ca}$ $(>43 \mathrm{mmol} / \mathrm{l})$ and $\mathrm{P}_{\mathrm{i}}(>33 \mathrm{mmol} / \mathrm{l})$. Black tea contained the highest concentration of $\mathrm{F}(1.63 \mathrm{mg} / \mathrm{l})$, whereas $\mathrm{F}$ concentration in other agents normally varied between 0 and $1 \mathrm{mg} / \mathrm{l}$.

Many of the test agents under study were undersaturated with respect to both HAP and FAP. Exceptionally, Henniez mineral water, Alcacyl 500 and Alka-Seltzer fizzy tablets, Kiwi and Slimline yogurts, coffee and teas (except for rose hip tea) were supersaturated with respect to both minerals. Valser mineral water, natural and forest berries yogurts, Thomy French Light salad dressing were undersaturated with respect to HAP but supersaturated with respect to FAP.

Table 3 shows the chemical properties with a significant impact on $\Delta \mathrm{SH}$ after a 2 and $4 \mathrm{~min}$ immersion in the multiple linear regression analysis. In this analysis, $52 \%$ of the variation of $\Delta \mathrm{SH}$ after $2 \mathrm{~min}$ immersion and $61 \%$ of the variation after 4 min immersion could be explained by $\mathrm{pH}$, buffering capacity, $\mathrm{Ca}$ and $\mathrm{F}$ concentrations $(P<0.05)$.

There were high bivariate correlations between $\Delta \mathrm{SH}$ and the $\mathrm{pH}$, the $(\mathrm{p} K-\mathrm{p} I)_{\mathrm{HAP}}$ and $(\mathrm{p} K-\mathrm{p} I)_{\mathrm{FAP}}$ (Table 4). However, the concentrations of $\mathrm{Ca}, \mathrm{P}_{\mathrm{i}}$ and $\mathrm{F}$, the titratable acidity and the buffering capacity showed small bivariate correlations with $\Delta \mathrm{SH}$.

\section{Discussion}

In agreement with previous studies ${ }^{(4,5,22,23)}$, the present study indicated that soft drinks, energy drinks (Red Bull), sports 
Table 2. Original surface hardness $\left(\mathrm{SH}_{\text {baseline}}\right)$ of specimens, and the changes within the first $2 \min \left(\Delta \mathrm{SH}_{2-0}=\mathrm{SH}_{2 \min }-\right.$ $\left.\mathrm{SH}_{\text {baseline }}\right)$ and the second $2 \min \left(\Delta \mathrm{SH}_{4-2}=\mathrm{SH}_{4 \min }-\mathrm{SH}_{2 \text { min }}\right)$ incubation in different dietary agents and medications (Mean values with their standard errors)

\begin{tabular}{|c|c|c|c|c|c|c|c|c|}
\hline & \multicolumn{2}{|c|}{$\mathrm{SH}_{\text {baseline }}$} & \multicolumn{2}{|c|}{$\Delta \mathrm{SH}_{2-0}$} & \multirow[b]{2}{*}{$P$} & \multicolumn{2}{|c|}{$\Delta \mathrm{SH}_{4-2}$} & \multirow[b]{2}{*}{$P$} \\
\hline & Mean & SEM & Mean & SEM & & Mean & SEM & \\
\hline \multicolumn{9}{|l|}{ Soft drinks } \\
\hline Carpe Diem Kombucha fresh & $526 \cdot 8$ & 6.5 & $-190 \cdot 2$ & $8 \cdot 0$ & $0.002^{\star}$ & -143.5 & $10 \cdot 4$ & $0.002 \dagger$ \\
\hline Coca-Cola & 513.4 & $7 \cdot 7$ & $-157 \cdot 4$ & $21 \cdot 1$ & $0.002^{*}$ & -153.3 & 15.9 & $0.002 \dagger$ \\
\hline Coca-Cola light & $600 \cdot 3$ & $16 \cdot 1$ & $-276 \cdot 7$ & $17 \cdot 0$ & $0.002^{*}$ & $-147 \cdot 0$ & $13 \cdot 8$ & $0.002 \dagger$ \\
\hline Fanta regular orange & $513 \cdot 3$ & $10 \cdot 0$ & $-244 \cdot 8$ & $12 \cdot 4$ & $0.002^{*}$ & $-136 \cdot 9$ & $7 \cdot 3$ & $0.002 \dagger$ \\
\hline Ice tea classic & $517 \cdot 0$ & 13.9 & -84.3 & $12 \cdot 6$ & $0.002^{*}$ & -99.3 & 14.9 & $0.002 \dagger$ \\
\hline Ice tea lemon & $511 \cdot 7$ & $6 \cdot 9$ & $-86 \cdot 1$ & $8 \cdot 0$ & $0.002^{\star}$ & $-106 \cdot 8$ & $8 \cdot 7$ & $0.002 \dagger$ \\
\hline Ice tea peach & $541 \cdot 0$ & $14 \cdot 8$ & -82.4 & 14.5 & $0.002^{*}$ & $-158 \cdot 7$ & 11.8 & $0.002 \dagger$ \\
\hline Pepsi Cola & $563 \cdot 3$ & $16 \cdot 9$ & $-190 \cdot 8$ & $12 \cdot 8$ & $0.002^{\star}$ & $-106 \cdot 8$ & $10 \cdot 7$ & $0.002 \dagger$ \\
\hline Pepsi Cola light & $512 \cdot 7$ & $7 \cdot 7$ & $-180 \cdot 4$ & $8 \cdot 0$ & $0.002^{*}$ & $-110 \cdot 0$ & $9 \cdot 2$ & $0.002 \dagger$ \\
\hline Rivella blue & $530 \cdot 2$ & $16 \cdot 4$ & $-253 \cdot 8$ & $17 \cdot 8$ & $0.002^{\star}$ & -138.9 & 5.6 & $0.002 \dagger$ \\
\hline Rivella green & $505 \cdot 6$ & $18 \cdot 3$ & -144.9 & $19 \cdot 4$ & $0.002^{*}$ & $-142 \cdot 7$ & $22 \cdot 6$ & $0.002 \dagger$ \\
\hline Rivella red & $532 \cdot 0$ & $16 \cdot 4$ & $-211 \cdot 1$ & $10 \cdot 8$ & $0.002^{*}$ & $-136 \cdot 0$ & 10.9 & $0.002 \dagger$ \\
\hline Sinalco & 514.3 & $4 \cdot 8$ & $-166 \cdot 5$ & $7 \cdot 8$ & $0.002^{*}$ & $-120 \cdot 5$ & $9 \cdot 8$ & $0.002 \dagger$ \\
\hline Sprite & $513 \cdot 2$ & 12.9 & $-192 \cdot 9$ & $19 \cdot 6$ & $0.002^{*}$ & -119.5 & 11.9 & $0.002 \dagger$ \\
\hline \multicolumn{9}{|l|}{ Energy drink } \\
\hline Red Bull & 534.9 & $18 \cdot 7$ & -88.7 & 22.5 & $0.009^{\star}$ & $-135 \cdot 7$ & $10 \cdot 2$ & $0.002 \dagger$ \\
\hline \multicolumn{9}{|l|}{ Sports drinks } \\
\hline Gatorade & $513 \cdot 2$ & $7 \cdot 3$ & $-124 \cdot 7$ & $9 \cdot 3$ & $0.002^{*}$ & -107.5 & $13 \cdot 6$ & $0.002 \dagger$ \\
\hline Isostar & 539.9 & $11 \cdot 1$ & -35.4 & 5.5 & $0.002^{*}$ & -0.2 & 7.6 & 1.000 \\
\hline Powerade & $510 \cdot 0$ & $6 \cdot 3$ & $-62 \cdot 7$ & $6 \cdot 0$ & $0.002^{\star}$ & $-96 \cdot 3$ & 11.9 & $0.002 \dagger$ \\
\hline \multicolumn{9}{|l|}{ Juice } \\
\hline Apple juice & $560 \cdot 9$ & 26.5 & -145.4 & $13 \cdot 6$ & $0.004^{*}$ & $-145 \cdot 8$ & $16 \cdot 1$ & $0.002 \dagger$ \\
\hline Carrot juice & 531.9 & 12.5 & $-13 \cdot 4$ & 5.5 & 0.037 & $-10 \cdot 5$ & 3.4 & 0.020 \\
\hline Grapefruit juice & 491.0 & 8.6 & $-152 \cdot 8$ & $7 \cdot 6$ & $0.002^{*}$ & 128.9 & $9 \cdot 7$ & $0.002 \dagger$ \\
\hline Orange juice, Del Monte & $500 \cdot 6$ & $6 \cdot 1$ & $-35 \cdot 2$ & 5.4 & $0.002^{\star}$ & $-72 \cdot 2$ & $8 \cdot 6$ & $0.002 \dagger$ \\
\hline Orange juice, Hohes C & $590 \cdot 8$ & 11.8 & $-59 \cdot 8$ & 8.7 & $0.002^{*}$ & $-100 \cdot 3$ & $15 \cdot 0$ & $0.002 \dagger$ \\
\hline \multicolumn{9}{|l|}{ Fruit } \\
\hline Apricot & $519 \cdot 1$ & 5.9 & $-120 \cdot 3$ & $9 \cdot 2$ & $0.002^{*}$ & $-103 \cdot 6$ & 7.5 & $0.002 \dagger$ \\
\hline Kiwi & $499 \cdot 8$ & $6 \cdot 2$ & $-116 \cdot 8$ & $9 \cdot 2$ & $0.002^{*}$ & -110.3 & 6.4 & $0.002 \dagger$ \\
\hline Orange & $561 \cdot 2$ & $13 \cdot 1$ & -97.4 & $7 \cdot 2$ & $0.002^{*}$ & -101.9 & $14 \cdot 6$ & $0.002 \dagger$ \\
\hline \multicolumn{9}{|l|}{ Alcoholic drinks } \\
\hline Bacardi Breezer orange & $572 \cdot 1$ & $17 \cdot 0$ & -224.9 & $13 \cdot 3$ & $0.002^{*}$ & $-130 \cdot 0$ & 8.5 & $0.002 \dagger$ \\
\hline Cynar & $519 \cdot 1$ & 8.4 & +1.2 & 5.4 & 0.695 & $+9 \cdot 1$ & $7 \cdot 8$ & 0.275 \\
\hline Beer, Carlsberg & $511 \cdot 1$ & $10 \cdot 4$ & -1.6 & 5.5 & 1.000 & $-5 \cdot 0$ & 3.5 & 0.124 \\
\hline Beer, Eichhof & $520 \cdot 6$ & $10 \cdot 1$ & +0.5 & $8 \cdot 0$ & 0.846 & $-13 \cdot 8$ & $3 \cdot 6$ & $0.006 \dagger$ \\
\hline Champagner, Freixenet & $531 \cdot 3$ & 8.0 & -126.9 & $7 \cdot 3$ & $0.002^{*}$ & -98.8 & $10 \cdot 0$ & $0.002 \dagger$ \\
\hline Red wine, Collivo & 543.4 & $22 \cdot 3$ & $-31 \cdot 1$ & $5 \cdot 6$ & $0.002^{*}$ & -33.4 & $5 \cdot 6$ & $0.002 \dagger$ \\
\hline Red wine, Montagne & $556 \cdot 2$ & 12.5 & $-20 \cdot 5$ & 7.4 & 0.027 & $-35 \cdot 9$ & 11.4 & 0.557 \\
\hline White wine, La Côte & $505 \cdot 0$ & $8 \cdot 1$ & -24.9 & 3.9 & $0.002^{*}$ & $-38 \cdot 1$ & 5.5 & $0.002 \dagger$ \\
\hline Ice vodka, Smirnoff & $565 \cdot 3$ & $11 \cdot 1$ & $-173 \cdot 9$ & $9 \cdot 0$ & $0.002^{*}$ & $-132 \cdot 4$ & $10 \cdot 1$ & $0.002 \dagger$ \\
\hline Medications & & & & & & & & \\
\hline Alca-C fizzy tablet & 533.6 & $11 \cdot 3$ & $-13 \cdot 2$ & $5 \cdot 0$ & 0.037 & -7.5 & $6 \cdot 1$ & $0 \cdot 160$ \\
\hline Alcacyl 500 & $527 \cdot 8$ & 11.8 & $-2 \cdot 3$ & 5.5 & 0.492 & $+2 \cdot 7$ & 3.7 & 0.557 \\
\hline Alka-Seltzer fizzy tablet & $512 \cdot 3$ & $7 \cdot 7$ & -3.9 & $4 \cdot 8$ & 0.492 & $-15 \cdot 0$ & $4 \cdot 2$ & $0.009 \dagger$ \\
\hline Aspirine-C fizzy tablet & 534.6 & $7 \cdot 1$ & -17.4 & 4.7 & $0.006^{\star}$ & -8.4 & $3 \cdot 8$ & 0.049 \\
\hline Berocca fizzy tablet & 511.4 & $5 \cdot 3$ & -1.7 & $7 \cdot 0$ & 0.846 & $+1 \cdot 2$ & 4.4 & 0.846 \\
\hline Fluimucil 200 fizzy tablet & $530 \cdot 8$ & 9.5 & -9.4 & $9 \cdot 7$ & 0.322 & $-19 \cdot 1$ & 5.4 & $0.009 \dagger$ \\
\hline Neocitran & 541.5 & 13.3 & -249.5 & $10 \cdot 9$ & $0.002^{*}$ & $-119 \cdot 8$ & $5 \cdot 0$ & $0.002 \dagger$ \\
\hline Vitamin C fizzy tablet, Actilife & 509.5 & $8 \cdot 3$ & $-88 \cdot 2$ & $7 \cdot 1$ & $0.002^{*}$ & $-98 \cdot 6$ & 4.9 & $0.002 \dagger$ \\
\hline Vitamin C fizzy tablet, Streuli & $549 \cdot 4$ & 19.5 & $-139 \cdot 1$ & 11.3 & $0.002^{*}$ & $-147 \cdot 7$ & $8 \cdot 8$ & $0.002 \dagger$ \\
\hline Siccoral & $525 \cdot 7$ & $16 \cdot 6$ & -7.4 & $6 \cdot 0$ & 0.322 & $-21 \cdot 0$ & $6 \cdot 1$ & $0.002 \dagger$ \\
\hline Yogurt & & & & & & & & \\
\hline Kiwi Tropicana & $548 \cdot 3$ & $8 \cdot 7$ & $+7 \cdot 3$ & $10 \cdot 3$ & 0.770 & $+18 \cdot 1$ & $16 \cdot 1$ & 0.492 \\
\hline Nature & $524 \cdot 3$ & 4.3 & $+2 \cdot 8$ & 3.6 & 0.375 & -8.5 & $4 \cdot 3$ & 0.160 \\
\hline Slimline & $573 \cdot 7$ & 18.5 & $-3 \cdot 3$ & $10 \cdot 2$ & 0.105 & $-3 \cdot 0$ & $7 \cdot 1$ & 0.275 \\
\hline Forest berries & $525 \cdot 0$ & 8.7 & $-6 \cdot 3$ & $2 \cdot 7$ & 0.049 & $-5 \cdot 6$ & 1.8 & $0.009 \dagger$ \\
\hline Mineral water & & & & & & & & \\
\hline Henniez & 543.0 & 11.9 & $+3 \cdot 8$ & $10 \cdot 6$ & 1.000 & -9.5 & $5 \cdot 1$ & 0.131 \\
\hline Henniez sparkling & $501 \cdot 3$ & $7 \cdot 4$ & $-1 \cdot 0$ & $3 \cdot 2$ & 0.760 & +0.4 & $3 \cdot 1$ & 0.846 \\
\hline Valser & $491 \cdot 7$ & $5 \cdot 0$ & -1.5 & $2 \cdot 3$ & 0.625 & $-4 \cdot 3$ & $3 \cdot 1$ & 0.275 \\
\hline Valser Viva Lemon & $506 \cdot 3$ & 11.6 & $-81 \cdot 0$ & 10.9 & $0.002^{\star}$ & $-89 \cdot 7$ & $6 \cdot 7$ & $0.002 \dagger$ \\
\hline Tea & & & & & & & & \\
\hline Rose hip & 545.6 & $16 \cdot 2$ & $-181 \cdot 1$ & $19 \cdot 6$ & $0.006^{*}$ & $-117 \cdot 8$ & 5.9 & $0.006 \dagger$ \\
\hline Pepper mint & 519.5 & $6 \cdot 7$ & +0.8 & $5 \cdot 2$ & 0.922 & $+7 \cdot 2$ & $5 \cdot 0$ & 0.155 \\
\hline
\end{tabular}


Table 2. Continued

\begin{tabular}{|c|c|c|c|c|c|c|c|c|}
\hline & \multicolumn{2}{|c|}{$\mathrm{SH}_{\text {baseline }}$} & \multicolumn{2}{|c|}{$\Delta \mathrm{SH}_{2-0}$} & \multirow[b]{2}{*}{$P$} & \multicolumn{2}{|c|}{$\Delta \mathrm{SH}_{4-2}$} & \multirow[b]{2}{*}{$P$} \\
\hline & Mean & SEM & Mean & SEM & & Mean & SEM & \\
\hline Black tea & $507 \cdot 4$ & 8.5 & $-1 \cdot 2$ & $5 \cdot 7$ & 1.000 & $+2 \cdot 4$ & $2 \cdot 8$ & 0.625 \\
\hline Wild berries & $603 \cdot 7$ & 9.5 & $+2 \cdot 1$ & $7 \cdot 4$ & 0.846 & $+5 \cdot 3$ & $5 \cdot 3$ & 0.322 \\
\hline \multicolumn{9}{|l|}{ Coffee } \\
\hline Espresso & $516 \cdot 5$ & $7 \cdot 1$ & $+3 \cdot 7$ & $5 \cdot 0$ & 0.492 & +0.5 & $5 \cdot 8$ & 0.846 \\
\hline \multicolumn{9}{|l|}{ Salad dressing } \\
\hline Thomy French Classic & 548.6 & 9.5 & $-21 \cdot 2$ & $5 \cdot 8$ & $0.002^{*}$ & $-4 \cdot 0$ & $6 \cdot 7$ & 0.492 \\
\hline Thomy French Light & $509 \cdot 1$ & $14 \cdot 1$ & $-32 \cdot 6$ & $4 \cdot 0$ & $0.002^{*}$ & $-61 \cdot 4$ & 8.5 & $0.002 \dagger$ \\
\hline
\end{tabular}

drinks, juices, fruits, and some medications and alcoholic drinks caused statistically significant decrease in $\mathrm{SH}$ of enamel samples. Yogurts, teas, mineral waters and coffee, except for those that were flavoured with acidic additives, did not have a detrimental effect on enamel $\mathrm{SH}$.

The results highlight the role of acidic additives in increasing erosive capacity of potentially erosive agents. The fruitbased or other acidic flavourings added to 'plain' or 'flat' drinks and foods, which are intended to stimulate taste, contribute to lower acidity and, consequently, induce erosion. Yogurt is a good example for demonstrating the effect of acidic additives. Natural yogurt caused no erosion in spite of its low $\mathrm{pH}$ value (3.91). This can be attributed to its high $(\mathrm{p} K-\mathrm{p} I)_{\mathrm{HAP}}$ resulting from high concentrations of $\mathrm{Ca}$ and $\mathrm{P}_{\mathrm{i}}$. The addition of berries (forest berries yogurt) caused a clinically not relevant reduction in $\mathrm{SH}$ within the second 2 min exposure. Even though this brand had higher $\mathrm{Ca}$ and $\mathrm{P}_{\mathrm{i}}$ concentrations than natural yogurt, its $\mathrm{pH}$ of 3.77 was too low for it to be supersaturated with respect to HAP. These findings are in accordance with other studies ${ }^{(24,25)}$. Similarly, compared with plain mineral water or tea, flavoured products, such as Valser Viva Lemon mineral water and rose hip tea, had much lower $\mathrm{pH}$ and negative $(\mathrm{p} K-\mathrm{p} I)_{\mathrm{HAP}}$, and hence caused a statistically significant reduction in SH. Moreover, it has been suggested that fruit-based acids might enhance the buffering capacity $^{(12)}$, which perhaps explains the higher buffering capacity and titratable acidity for the flavoured liquids (Table 1). Therefore, the above-mentioned flavoured products, from the chemical composition point of view, should be classified as soft drinks. Their erosive potential would be expected to be much closer to erosive drinks than to plain products $^{(6,26)}$.

The $\mathrm{p} K-\mathrm{p} I$ with respect to tooth mineral, determined by the $\mathrm{pH}$ value and the concentrations of $\mathrm{Ca}, \mathrm{P}_{\mathrm{i}}$ and $\mathrm{F}$ in $\mathrm{a}$ solution, is the driving force for mineral dissolution. When $(\mathrm{p} K-\mathrm{p} I)_{\mathrm{HAP}}<0$, the solution is undersaturated with respect to HAP, which chemically and structurally resembles natural tooth $^{(27)}$. In acidic media, the value of the ion activity product for HAP was a good predictor of enamel lesion ${ }^{(28,29)}$. Therefore, this solution may induce demineralisation of the enamel. When $(\mathrm{p} K-\mathrm{p} I)_{\mathrm{HAP}}>0$, the solution is supersaturated, so favours remineralisation ${ }^{(30)}$. Previous studies have observed that $(\mathrm{p} K-\mathrm{p} I)_{\mathrm{HAP}}$ plays an important role in tooth dissolution. A small change in $(\mathrm{p} K-\mathrm{p} I)_{\mathrm{HAP}}$ might result in a marked difference in the dissolution rate of enamel ${ }^{(13,31,32)}$. As $(\mathrm{p} K-\mathrm{p} I)_{\mathrm{HAP}}$ is dependent on $\mathrm{pH}$ and $\mathrm{Ca}$ and $\mathrm{P}_{\mathrm{i}}$ concentrations, it was not included in the multiple regression analysis. However, there was a negative and strong bivariate correlation between both $(\mathrm{p} K-\mathrm{p} I)_{\mathrm{HAP}}$ and $(\mathrm{p} K-\mathrm{p} I)_{\mathrm{FAP}}$ and $\Delta \mathrm{SH}$ after both 2 and $4 \mathrm{~min}$. In general, bivariate analyses may be misleading because possible interactions between variables are neglected. Interestingly, the concentrations of $\mathrm{Ca}, \mathrm{P}_{\mathrm{i}}$ and $\mathrm{F}$ alone had a weak correlation with $\Delta \mathrm{SH}[0]$, whereas the $\mathrm{p} K-\mathrm{p} I$ defined by the combination of these variables (and the $\mathrm{pH}$ ) showed a strong correlation.

Many studies have demonstrated that $\mathrm{pH}$ is a good predictor of dental erosion: as the $\mathrm{pH}$ of the investigated product decreases, there is an increased amount of erosion, independent of the way in which erosion is measured ${ }^{(22,33)}$. The buffer properties (buffering capacity or titratable acidity) have also been considered to be important ${ }^{(5)}$, even more than $\mathrm{pH}^{(34,35)}$, in predicting the erosive potential because it maintains the $\mathrm{H}^{+}$concentration available for the interaction with the tooth surface ${ }^{(1)}$. The effect of buffering might, however, vary with $\mathrm{pH}$. Because erosive demineralisation takes place at least partly beneath the enamel surface, buffering capacity may become increasingly important as $\mathrm{pH}$ falls, since this is accompanied by an increase in dissolution rate. Consequently, while diffusion may be capable of supplying sufficient $\mathrm{H}^{+}$ions at higher $\mathrm{pH}$ (slow dissolution), increased buffering will be required at lower $\mathrm{pH}$ in order to maintain the supply of $\mathrm{H}^{+}$ions ${ }^{(36)}$. However, the relative importance of $\mathrm{pH}$ and buffering properties could depend on factors such as exposure time and the ratio of the volume of solution to the area of exposed tooth surface. In an in vitro study using a low ratio of solution to specimen area, Jensdottir et al. ${ }^{(23)}$ reported a significant correlation between buffer properties, titratable acidity, buffering capacity and tooth tissue dissolution after exposure to selected soft drinks for a long time $(24 \mathrm{~h})$, while after a short-term exposure $(3 \mathrm{~min})$, erosion was associated with $\mathrm{pH}$ but not with titratable acidity ${ }^{(37)}$. They speculated, therefore, that titratable acidity was the better predictor of erosive potential during longer erosive challenges and $\mathrm{pH}$ was better for short challenges. However, Hara \& Zero ${ }^{(7)}$ observed that after $2 \mathrm{~h}$ exposure, titratable acidity showed a low-to-moderate correlation with enamel demineralisation, while $\mathrm{pH}$ value was the best predictor for erosion. They ascribed this result to the relatively high 
Table 3. Multiple linear regression analysis of the changes in surface hardness $(\Delta \mathrm{SH})$ of all specimens after immersion in all agents for 2 and $4 \mathrm{~min}^{\star}$ ( $\beta$ Coefficients)

\begin{tabular}{|c|c|c|c|c|c|c|c|c|c|c|}
\hline \multirow[b]{2}{*}{$\Delta \mathrm{SH}$} & \multicolumn{2}{|c|}{$\mathrm{pH}$} & \multicolumn{2}{|c|}{ Buffering capacity } & \multicolumn{2}{|c|}{ Ca concentration } & \multicolumn{2}{|c|}{ F concentration } & \multicolumn{2}{|c|}{ Intercept } \\
\hline & $P$ & $\beta$ & $P$ & $\beta$ & $P$ & $\beta$ & $P$ & $\beta$ & $P$ & $\beta$ \\
\hline $\begin{array}{l}\Delta \mathrm{SH}_{2-0}\left(R^{2} 0.52\right) \dagger \\
\Delta \mathrm{SH}_{4-0}\left(R^{2} 0.61\right) \ddagger\end{array}$ & $\begin{array}{l}<0.0001 \\
<0.0001\end{array}$ & $\begin{array}{l}-46.5 \\
-81.0\end{array}$ & $\begin{array}{l}<0.0001 \\
<0.0001\end{array}$ & $\begin{array}{l}-0.5 \\
-0.6\end{array}$ & $\begin{array}{l}<0.0001 \\
<0.0001\end{array}$ & $\begin{array}{l}-1 \cdot 2 \\
-2 \cdot 6\end{array}$ & $\begin{array}{l}0.0006 \\
0.0055\end{array}$ & $\begin{array}{l}-34.2 \\
-39.9\end{array}$ & $\begin{array}{l}<0.0001 \\
<0.0001\end{array}$ & $\begin{array}{l}300 \cdot 3 \\
521 \cdot 1\end{array}$ \\
\hline
\end{tabular}

${ }^{*} P$ values $(\beta$ : estimate) are listed for those variables with a significant impact on $\Delta \mathrm{SH}$.

$+\Delta \mathrm{SH}_{2-0}=\mathrm{SH}_{2 \text { min }}-\mathrm{SH}_{\text {baseline }}$.

$\ddagger \Delta \mathrm{SH}_{4-0}=\mathrm{SH}_{4 \text { min }}-\mathrm{SH}_{\text {baseline. }}$.

volume $(30 \mathrm{ml})$ used in their study. Buffering properties are likely to be relatively more important when a low volume of solution is used, as the $\mathrm{pH}$ would be raised more easily by mineral dissolution ${ }^{(7)}$. The dependence of tooth erosion on both $\mathrm{pH}$ value and buffering capacity observed in the present study, and the lack of a significant effect of titratable acidity could thus be due to our use of short erosive challenges and an adequate, well-stirred volume of the test product.

The literature is contradictory with regard to the erosive potential of acidic drinks and foods containing $\mathrm{F}^{(4,5,22)}$. Previous studies have shown that the erosive capacity of different drinks was significantly and negatively associated with their original $\mathrm{F}$ concentration ${ }^{(4,5)}$. This observation was confirmed in the present study. In contrast, Larsen \& Nyvad $^{(22)}$ reported that $\mathrm{F}$ concentration in eighteen soft drinks had no effect on the depth of tooth erosion. Furthermore, a study by Larsen \& Richards ${ }^{(38)}$ showed that in drinks with $\mathrm{pH}$ above $3, \mathrm{~F}$ concentrations reduced the in vitro development of erosion by $28 \%$; in drinks with $\mathrm{pH}$ below 3, erosion was not affected, despite total $\mathrm{F}$ concentrations of 20 parts per million and saturation with calcium fluoride. It is worth noting that in those studies, severe acid attacks with surface loss was chosen, while in the present study initial erosion (softening) caused by various agents was assessed.

A higher concentration of the $\mathrm{Ca}$ and/or $\mathrm{P}_{\mathrm{i}}$ in a solution will increase the $\mathrm{p} K-\mathrm{p} I$ with respect to dental mineral, so that the presence of suitable concentrations of $\mathrm{Ca}$ and $\mathrm{P}_{\mathrm{i}}$ may counteract tooth erosion caused by acidic drinks and foods. Some studies have proved that lower levels of enamel demineralisation were found in Ca-containing drinks than in those without $\mathrm{Ca}^{(23,39-41)}$. The relatively higher concentrations of $\mathrm{Ca}$ and $\mathrm{P}_{\mathrm{i}}$ are most probably responsible for the less erosive effect of Isostar compared with other sports drinks. Isostar does not contain other protective ingredients, such as casein. The results of multiple linear regression analyses indicated a significant relationship between $\mathrm{Ca}$ concentration and erosion. However, there was no evidence of a relationship between $\mathrm{P}_{\mathrm{i}}$ and tooth erosion. There are four species of inorganic $\mathrm{P}_{\mathrm{i}}$, namely $\mathrm{H}_{3} \mathrm{PO}_{4}$, $\mathrm{H}_{2} \mathrm{PO}_{4}^{-}, \mathrm{HPO}_{4}^{2-}$ and $\mathrm{PO}_{4}^{3-}$, in a given solution and their proportions depend on the $\mathrm{pH}^{(42)}$. At the $\mathrm{pH}$ of erosive drinks (approximately 2-4), only a minute fraction of the total $\mathrm{P}_{\mathrm{i}}$ (of the order of $10^{-13}$ ) is in the form of $\mathrm{PO}_{4}^{3-}$ ions ${ }^{(42)}$, which are the only important $\mathrm{P}_{\mathrm{i}}$ species in the ion activity product of HAP and FAP. Therefore, enormous quantities of $\mathrm{P}_{\mathrm{i}}$ are required to raise the degree of saturation of the solution. This may be the reason why $\mathrm{P}_{\mathrm{i}}$ is ineffective in the present study.
The formation of a pellicle with human saliva as well as the exposure time scale of a few minutes used in the present study is of particular physiological relevance and clinical interest. First, this exposure time is comparable with clearance time of acids in the mouth ${ }^{(23)}$. Second, in the early stage, acids diffuse into the tooth and remove $\mathrm{Ca}$ and $\mathrm{P}_{\mathrm{i}}$ from the outer few micrometres of hard tissues, forming a demineralised, weakened layer. Remineralisation is possible in this stage, since the remaining enamel can serve as framework in which minerals can be deposited again ${ }^{(43)}$.

The present in vitro study, however, cannot totally reproduce the clinical conditions, and should only be interpreted as a prediction of the relative erosive potential of a dietary substance or a medication. Erosion is a multifactorial condition, and its occurrence and development depend on many risk and protective factors as well as on their interplay $^{(44)}$. In addition to the erosive potential of dietary substances and medications, a variety of factors, for example frequency of acid intake, individual dietary habits (sipping, gulping, frothing or use of a straw) ${ }^{(45)}$, the physical properties (the adhesiveness and displacement) of these agents ${ }^{(46)}$, the flow rate, composition and clearing capability of the saliva, may influence the progress of tooth erosion ${ }^{(47)}$. However, an investigation of the parameters associated with the erosive potential of dietary substances and medications could act as a significant screening test through which dentists can provide instructional recommendations for patients at high risk of dental erosion. In addition, the present study covered a wide range of tested agents with various chemical and physical properties. Some components in these agents may have an

Table 4. Spearman's correlation coefficients: all chemical properties $v$. the changes in surface hardness $(\Delta \mathrm{SH})$ and the respective $P$ values

\begin{tabular}{|c|c|c|c|c|}
\hline \multirow[b]{2}{*}{ Chemical property } & \multicolumn{2}{|c|}{$\Delta \mathrm{SH}_{2-0^{*}}$} & \multicolumn{2}{|c|}{$\Delta \mathrm{SH}_{4-0} \dagger$} \\
\hline & $R^{2}$ & $P$ & $R^{2}$ & $P$ \\
\hline $\mathrm{pH}$ & -0.83 & $<0.0001$ & -0.86 & $<0.0001$ \\
\hline Titratable acidity & 0.14 & 0.0006 & 0.16 & 0.0001 \\
\hline Buffering capacity & 0.04 & 0.321 & 0.06 & 0.154 \\
\hline Ca concentration & -0.27 & $<0.0001$ & -0.28 & $<0.0001$ \\
\hline $\mathrm{P}_{\mathrm{i}}$ concentration & -0.13 & 0.0009 & -0.14 & 0.0005 \\
\hline F concentration & -0.11 & 0.006 & -0.11 & 0.005 \\
\hline$(\mathrm{pK}-\mathrm{p} /)_{\text {HAP }} \ddagger$ & -0.75 & $<0.0001$ & -0.78 & $<0.0001$ \\
\hline$(\mathrm{p} K-\mathrm{p} /)_{\mathrm{FAP}} \S$ & -0.70 & $<0.0001$ & -0.73 & $<0.0001$ \\
\hline
\end{tabular}

$\mathrm{pK}-\mathrm{pl}$, degree of saturation; HAP, hydroxyapatite; FAP, fluorapatite.

${ }^{*} \Delta \mathrm{SH}_{2-0}=\mathrm{SH}_{2 \text { min }}-\mathrm{SH}_{\text {baseline. }}$

$\dagger \Delta \mathrm{SH}_{4-0}=\mathrm{SH}_{4 \text { min }}-\mathrm{SH}_{\text {baseline }}$

$\ddagger \mathrm{pK}-\mathrm{p} /$ with respect to HAP.

$\S \mathrm{pK}-\mathrm{p} /$ with respect to FAP. 
influence on salivary pellicle and thus interfere in the correct assessment of tooth erosion. For example, black tea and red wine have been shown to have a profound effect on in vitro pellicle maturation, causing thickened layers of stained material to build up, which were not readily removed. The mechanism behind this effect was ascribed to the polyphenols contained $^{(48)}$. Salivary proline-rich proteins, particularly basic proline-rich proteins, via the proline rings ${ }^{(49)}$, have a particularly high affinity for dietary polyphenols ${ }^{(50,51)}$, as do histatins ${ }^{(52,53)}$.

In conclusion, the present study confirmed the erosive potential of a wide range of dietary substances and medications. Tooth erosion had a significant relationship with $\mathrm{pH}$, with buffering capacity, $\mathrm{F}$ and $\mathrm{Ca}$ concentrations. The degree of saturation with respect to HAP and FAP, illustrating the combined effect of these parameters, showed a high bivariate correlation with tooth erosion.

\section{Acknowledgements}

The present study was supported by a grant from the Swiss Society of Odontology (project no. 222-05). A. L. designed the protocol. B. M. conducted the experiments. X. W., R. P. S. and A. L. analysed the data and wrote the manuscript. A. L. had primary responsibility for the final content. All authors read and approved the final manuscript. None of the authors reported a conflict of interest. We thank Stefanie Hayoz, Institute of Mathematical Statistics and Actuarial Science, University of Bern, for the statistical analysis, and also Thiago Saads Carvalho, Faculdade de Odontologia da Universidade de São Paulo, for the help in the revision of this manuscript.

\section{References}

1. Zero DT (1996) Etiology of dental erosion-extrinsic factors. Eur J Oral Sci 104, 162-177.

2. Dugmore CR \& Rock WP (2003) Awareness of tooth erosion in 12 year old children and primary care dental practitioners. Community Dent Health 20, 223-227.

3. Ehlen LA, Marshall TA, Qian F, et al. (2008) Acidic beverages increase the risk of in vitro tooth erosion. Nutr Res $\mathbf{2 8}$, 299-303.

4. Lussi A, Jaggi T \& Scharer S (1993) The influence of different factors on in vitro enamel erosion. Caries Res 27, 387-393.

5. Lussi A, Jaeggi T \& Jaeggi-Scharer S (1995) Prediction of the erosive potential of some beverages. Caries Res 29, 349-354.

6. Parry J, Shaw L, Arnaud MJ, et al. (2001) Investigation of mineral waters and soft drinks in relation to dental erosion. J Oral Rehabil 28, 766-772.

7. Hara AT \& Zero DT (2008) Analysis of the erosive potential of calcium-containing acidic beverages. Eur J Oral Sci 116, 60-65.

8. Phelan J \& Rees J (2003) The erosive potential of some herbal teas. J Dent 31, 241-246.

9. Packer CD (2009) Cola-induced hypokalaemia: a super-sized problem. Int J Clin Pract 63, 833-835.

10. Jager DH, Vieira AM, Ruben JL, et al. (2008) Influence of beverage composition on the results of erosive potential measurement by different measurement techniques. Caries Res 42, 98-104.
11. Owens BM (2007) The potential effects of $\mathrm{pH}$ and buffering capacity on dental erosion. Gen Dent 55, 527-531.

12. Edwards M, Creanor SL, Foye RH, et al. (1999) Buffering capacities of soft drinks: the potential influence on dental erosion. J Oral Rehabil 26, 923-927.

13. Gray JA (1962) Kinetics of the dissolution of human dental enamel in acid. $J$ Dent Res 41, 633-645.

14. Featherstone JD \& Rodgers BE (1981) Effect of acetic, lactic and other organic acids on the formation of artificial carious lesions. Caries Res 15, 377-385.

15. Zero DT, Rahbek I, Fu J, et al. (1990) Comparison of the iodide permeability test, the surface microhardness test, and mineral dissolution of bovine enamel following acid challenge. Caries Res 24, 181-188.

16. Chen PS, Toribara TY \& Warner H (1956) Microdetermination of phosphorus. Anal Chem 28, 1756-1758.

17. Larsen MJ (1986) An investigation of the theoretical background for the stability of the calcium-phosphate salts and their mutual conversion in aqueous solutions. Arch Oral Biol 31, 757-761.

18. McDowell H, Gregory TM \& Brown E (1977) Solubility of $\mathrm{Ca}_{5}\left(\mathrm{PO}_{4}\right)_{3} \mathrm{OH}$ in the system $\mathrm{Ca}(\mathrm{OH})_{2}-\mathrm{H}_{3} \mathrm{PO}_{4}-\mathrm{H}_{2} \mathrm{O}$ at 5,25 and $37^{\circ} \mathrm{C}$. J Res Natl Bur Stand $\mathbf{8 1}, 273-281$.

19. McCann HG (1968) The solubility of fluorapatite and its relationship to that of calcium fluoride. Arch Oral Biol 13, 987-1001.

20. Stiefel DJ (1976) Characteristics of an in vitro dental pellicle. $J$ Dent Res 55, 66-73.

21. Wetton S, Hughes J, West N, et al. (2006) Exposure time of enamel and dentine to saliva for protection against erosion: a study in vitro. Caries Res 40, 213-217.

22. Larsen MJ \& Nyvad B (1999) Enamel erosion by some soft drinks and orange juices relative to their $\mathrm{pH}$, buffering effect and contents of calcium phosphate. Caries Res 33, 81-87.

23. Jensdottir T, Bardow A \& Holbrook P (2005) Properties and modification of soft drinks in relation to their erosive potential in vitro. J Dent 33, 569-575.

24. Caglar E, Lussi A, Kargul B, et al. (2006) Fruit yogurt: any erosive potential regarding teeth? Quintessence Int 37, 647-651.

25. Lussi A \& Jaeggi $\mathrm{T}$ (2006) Chemical factors. In Dental Erosion: from Diagnosis to Therapy, pp. 77-87 [A Lussi, editor]. Basel: Karger.

26. Brown CJ, Smith G, Shaw L, et al. (2006) The erosive potential of flavoured sparkling water drinks. Int J Paediatr Dent 17, 86-91.

27. Yamagishi K, Onuma K, Suzuki T, et al. (2005) Materials chemistry: a synthetic enamel for rapid tooth repair. Nature 433, 819

28. Shellis RP, Wahab FK \& Heywood BR (1993) The hydroxyapatite ion activity product in acid solutions equilibrated with human enamel at 37 degrees C. Caries Res 27, 365-372.

29. Shellis RP (1996) A scanning electron-microscopic study of solubility variations in human enamel and dentine. Arch Oral Biol 41, 473-484.

30. Lussi A \& Jaeggi T (2008) Erosion - diagnosis and risk factors. Clin Oral Invest 12, S5-S13.

31. Margolis HC, Zhang YP, Lee CY, et al. (1999) Kinetics of enamel demineralization in vitro. J Dent Res 78, 1326-1335.

32. Tanaka M \& Kadoma Y (2000) Comparative reduction of enamel demineralization by calcium and phosphate in vitro. Caries Res 34, 241-245.

33. Mistry M \& Grenby TH (1993) Erosion by soft drinks of rat molar teeth assessed by digital image analysis. Caries Res 27, 21-25. 
34. Grobler SR, Senekal PJ \& Laubscher JA (1990) In vitro demineralization of enamel by orange juice, apple juice, Pepsi Cola and Diet Pepsi Cola. Clin Prev Dent 12, 5-9.

35. Milosevic A (1997) Sports drinks hazard to teeth. Br J Sports Med 31, 28-30.

36. Shellis RP, Barbour ME, Jones SB, et al. (2010) Effects of pH and acid concentration on erosive dissolution of enamel, dentine, and compressed hydroxyapatite. Eur J Oral Sci 118, 475-482.

37. Jensdottir T, Holbrook P, Nauntofte B, et al. (2006) Immediate erosive potential of cola drinks and orange juices. $J$ Dent Res 85, 226-230.

38. Larsen MJ \& Richards A (2002) Fluoride is unable to reduce dental erosion from soft drinks. Caries Res 36, 75-80.

39. West NX, Hughes JA, Parker DM, et al. (2003) Development of low erosive carbonated fruit drinks 2. Evaluation of an experimental carbonated blackcurrant drink compared to a conventional carbonated drink. J Dent 31, 361-365.

40. West NX, Hughes JA, Parker DM, et al. (1999) Development and evaluation of a low erosive blackcurrant juice drink. 2 . Comparison with a conventional blackcurrant juice drink and orange juice. J Dent 27, 341-344.

41. Hughes JA, West NX, Parker DM, et al. (1999) Development and evaluation of a low erosive blackcurrant juice drink in vitro and in situ. 1. Comparison with orange juice. $J$ Dent 27, 285-289.

42. Dawes $\mathrm{C}$ (2003) What is the critical $\mathrm{pH}$ and why does a tooth dissolve in acid? J Can Dent Assoc 69, 722-724.

43. Lussi A (2006) Erosive tooth wear - a multifactorial condition of growing concern and increasing knowledge.
In Dental Erosion: from Diagnosis to Therapy, pp. 1-8 [A Lussi, editor]. Basel: Karger.

44. Lussi A, Hellwig E, Zero D, et al. (2006) Erosive tooth wear: diagnosis, risk factors and prevention. Am J Dent 19, 319-325.

45. Zero D \& Lussi A (2006) Behavioral factors. In Dental Erosion: from Diagnosis to Therapy, pp. 100-105 [A Lussi, editor]. Basel: Karger.

46. Ireland AJ, McGuinness N \& Sherriff M (1995) An investigation into the ability of soft drinks to adhere to enamel. Caries Res 29, 470-476.

47. Hara AT, Lussi A \& Zero DT (2006) Biological factors. In Dental Erosion: from Diagnosis to Therapy, pp. 88-99 [A Lussi, editor]. Basel: Karger.

48. Joiner A, Muller D, Elofsson UM, et al. (2003) Adsorption from black tea and red wine onto in vitro salivary pellicles studied by ellipsometry. Eur J Oral Sci 111, 417-422.

49. Williamson MP (1994) The structure and function of prolinerich regions in proteins. Biochem J 297, 249-260.

50. Hagerman AE \& Butler LG (1981) The specificity of proanthocyanidin-protein interactions. J Biol Chem 256, 4494-4497.

51. Lu Y \& Bennick A (1998) Interaction of tannin with human salivary proline-rich proteins. Arch Oral Biol 43, 717-728.

52. Yan Q \& Bennick A (1995) Identification of histatins as tannin-binding proteins in human saliva. Biochem $J$ 311, 341-347.

53. Wroblewski K, Muhandiram R, Chakrabartty A, et al. (2001) The molecular interaction of human salivary histatins with polyphenolic compounds. Eur J Biochem 268, 4384-4397. 